\title{
EPISIOTOMIA VERSUS HUMANIZAÇÃO DA ASSISTÊNCIA DE ENFERMAGEM: IMPLICAÇÕES À SAÚDE DA MULHER
}

\author{
EPISIOTOMY VERSUS HUMANIZATION OF NURSING CARE: \\ WOMEN'S HEALTH IMPLICATIONS
}

\author{
Jessica Pereira Soares Alves ${ }^{1}$ \\ Edilson Salvador da Silva ${ }^{2}$ \\ Jozilane Pereira Soares ${ }^{3}$ \\ Raquel de Negreiros Moreira Silva ${ }^{4}$
}

RESUMO: Objetivo: Compreender os impactos causados pela Episiotomia e como o cuidado humanizado pode potencializar o processo de restauração da saúde da mulher. Método: Trata- se de um estudo bibliográfico com fontes publicadas em livros, periódicos, dissertações e artigos disponíveis em meios eletrônicos, sendo selecionados 20 artigos compreendidos no período de 2009 a 2019. Resultados: A episiotomia consiste em um procedimento operatório invasivo, mais recorrente na obstetrícia moderna, que interfere diretamente na qualidade de vida da mulher. Os estudos demonstram a omissão de informações, indicações, vantagens, desvantagens, possíveis complicações de sua prática, e o não consentimento prévio das mulheres, evidenciando a relação autoritária, exercida pelos profissionais durante a assistência ao parto. Conclusão: A humanização da assistência de enfermagem se faz necessária, quando se compreende os impactos da Episiotomia e as implicações que esta prática pode repercutir na vida da mulher.

Descritores: Humanização. Assistência de Enfermagem. Episiotomia. Saúde da Mulher.

ABSTRACT: Objective: To understand the impacts caused by Episiotomy and how humanized care can enhance the process of restoring women's health. Method: This is a bibliographic study with sources published in books, journals, dissertations and articles available in electronic means, being selected 20 articles from 2009 to 2019.

\footnotetext{
1 Discente no curso de Bacharelado em Enfermagem-UNESC FACULDADES. E-mail: jessica_p.s.a@hotmail.com.

2 Discente no curso de Bacharelado em Enfermagem-UNESC FACULDADES.

${ }^{3}$ Discente no curso de Bacharelado em Enfermagem-UNESC FACULDADES.

${ }^{4}$ Enfermeira, Mestre em Modelos de Decisão e Saúde-UFPB, Docente do Curso de Bacharelado em Enfermagem-UNESC FACULDADES. E-mail: kel.dudunana@gmail.com.
} 
Results: Episiotomy is an invasive surgical procedure, more recurrent in modern obstetrics, which directly interferes with women's quality of life. The studies show the omission of information, indications, advantages, disadvantages, possible complications of their practice, and the previous non-consent of women, evidencing the authoritarian relationship exercised by professionals during the delivery care. Conclusion: The humanization of nursing care is necessary, when we understand the impacts of Episiotomy and the implications that this practice may have on women's lives.

Descriptors: Humanization. Nursing care. Episiotomy. Women's health. 


\section{INTRODUÇÃO}

O parto é um processo fisiológico caracterizado pelo término da gestação e o nascimento do feto. Neste momento algumas condutas são importantes com a finalidade de promover um parto e nascimento saudável (PINHEIRO et al., 2012). Segundo Aratani et al (2014), o parto normal é considerado fisiológico porque o feto ultrapassa as barreiras do sistema reprodutor feminino sistematicamente por meio das contrações uterinas, proporcionando a expulsão da criança sem intervenções cirúrgicas, enquanto que, no parto cesariano, é necessário uma incisão na parede abdominal para a extração manual do bebê.

Embora as modificações fisiológicas sejam necessárias para que ocorra o parto, o assoalho pélvico fica exposto a modificações que poderão evoluir para lesões na região perineal. Assim, durante a expulsão do feto surge a possibilidade da realização da episiotomia (COSTA et al., 2011). Este é um procedimento cirúrgico (incisão), realizado no período expulsivo, que tem como objetivo evitar lacerações na região perineal, mas que, em algumas situações pode influenciar na ocorrência de um trauma mais grave que uma laceração espontânea (MATHIAS et al., 2015).

Para o Ministério da Saúde (2014) a episiotomia é defendida como indicação obstétrica para impedir ou diminuir a lesão dos tecidos do canal do parto, favorecer a liberação do feto, evitar danos desnecessários ao assoalho pélvico, visando evitar prolapsos genitais e incontinência urinária futura, além de reduzir o risco de morbimortalidade infantil, retocele, cistocele e relaxamento da musculatura pélvica. No entanto, os supostos efeitos adversos da episiotomia, estão relacionadas a extensão do corte com lesão de esfíncter anal e retal, resultados anatômicos não satisfatórios tais como pregas cutâneas, assimetria ou estreitamento excessivo do intróito, prolapso vaginal, fístula reto-vaginal e fístula anal, aumento na perda sanguínea e hematomas, dor, edema locais, infecção, deiscência e disfunção sexual. 
De acordo com o Manual da Maternidade Segura da Organização Mundial de Saúde (1996), a episiotomia é classificada como uma prática frequentemente utilizada de modo inadequado, podendo ser indicada ocasionalmente e com limitações, pois existem evidências de que seu uso rotineiro pode causar danos, sugerindo uma taxa ideal em torno de 10,0\%, porém é executada em $90 \%$ dos partos normais realizados em ambientes hospitalares no Brasil (2011).

A lei do exercício profissional 7.498/86 e o decreto-lei 94.406/87 assegura ao enfermeiro obstetra, realizar a assistência a parturiente, destacando a responsabilidade na articulação do cuidado humanizado, por meio de capacitação técnica e conhecimento científico que pode contribuir consideravelmente na transformação dos paradigmas atuais (POMPEU et al., 2017).

A equipe de saúde deve estar preparada para acolher a grávida, seu companheiro e família, respeitando todos os significados desse momento (GOMES et al.,2014). A assistência humanizada ao parto é muito discutida por enfermeiros obstetras, onde existe uma preocupação em instituir tecnologias que auxiliem na fisiologia do parto preservando a integridade corporal e evitando intervenções desnecessárias (SALGE et al., 2012).

Com base nesse estudo, estabeleceu-se os seguintes questionamentos: Qual a real necessidade de indicação da episiotomia, quais as possíveis implicações esta prática pode desencadear, e Como oferecer uma assistência humanizada a essas mulheres? Assim, a pesquisa tem como objetivo, encontrar artigos publicados a respeito de evidências em episiotomia e as implicações que este procedimento pode desencadear na vida da mulher, e como fornecer subsídios aos profissionais de enfermagem, a respeito de ações que garantam uma assistência humanizada e menos intervencionista.

\section{MÉTODO}

Buscou-se uma revisão sistemática da literatura, com buscas de estudos primários em grandes bases de dados: MEDLINE, LILACS, SCIELO, IBES, 
EMBASE, GOOGLE SCHOLAR com os seguintes descritores: Humanização, Assistência de Enfermagem, Episiotomia, Saúde da mulher. Os descritores fazem parte do banco de dados dos Descritores de Ciências da Saúde - DECS.

Identificou-se na primeira análise um número de 523 artigos. Tais estudos passaram por dois revisores e analisados quanto aos critérios de inclusão e exclusão. Os critérios de inclusão foram: estudos na língua inglesa ou portuguesa; publicados a menos de dez anos; artigos primários; amostra consistente em processo parturitivo. Os critérios de exclusão foram aqueles que não atenderem os critérios anteriores.

Após a consolidação e concordância dos estudos escolhidos pelos revisores, encontrou-se o número final de estudos elegíveis e que fizeram parte desta revisão.

\section{RESULTADOS E DISCUSSÃO}

O fluxograma das etapas de busca e seleção das publicações nas bases de dados foi sumarizada pelo algoritmo exposto na Figura 1. 
Figura 1 Algoritmo de pesquisa da revisão sistemática.
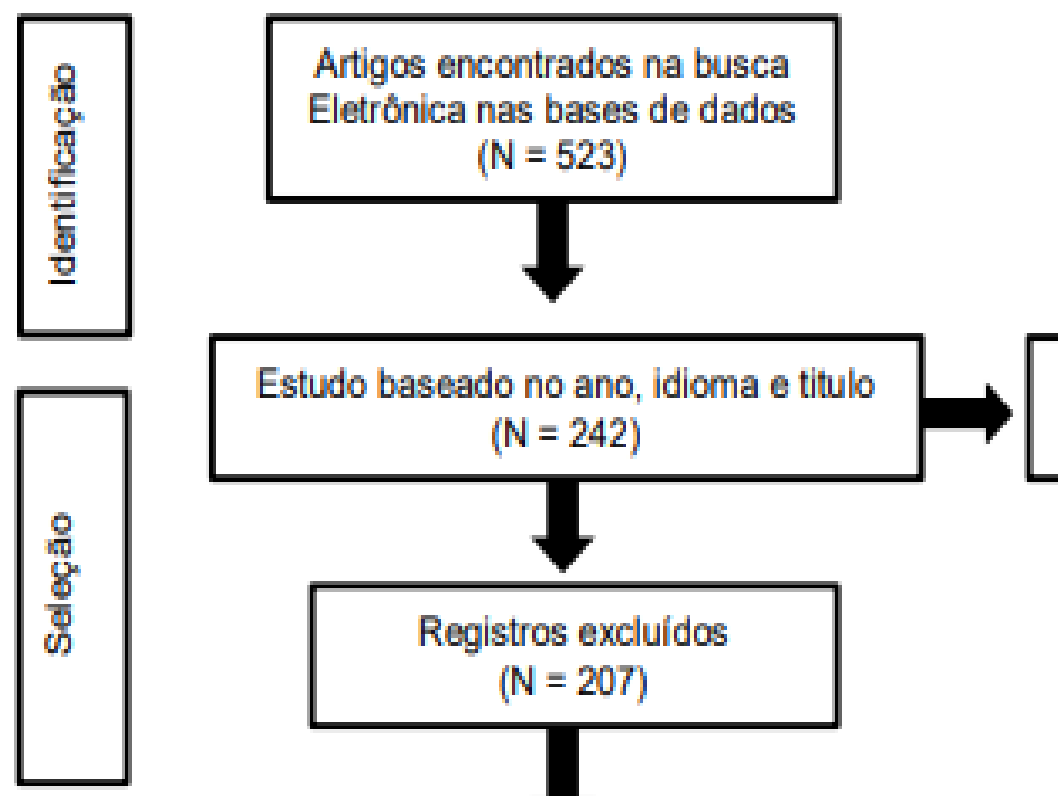

Artigos em duplicidade $(\mathrm{N}=14)$
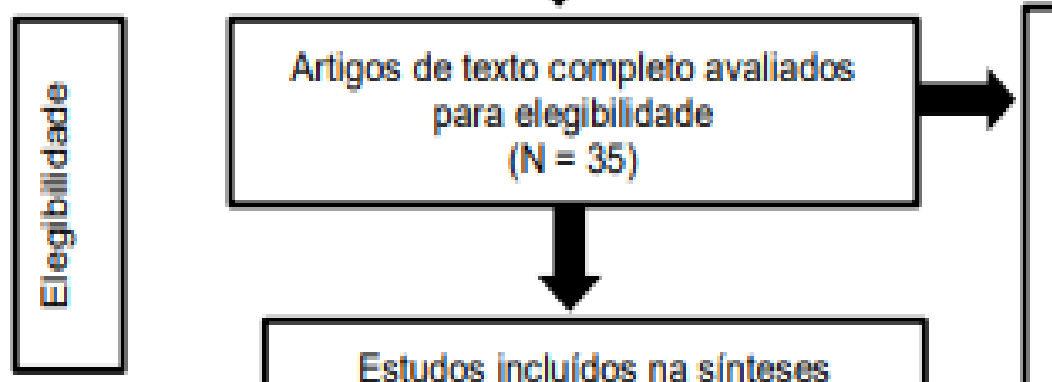

Registros excluidos com razōes $(\mathrm{N}=15)$

Outros $=10$

Artigos em discordåncia $=01$ Avaliaçăo de resumo $=04$

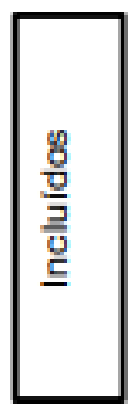

\section{Estudos incluidos na sinteses}

Qualitativa

$(\mathrm{N}=20)$

Avaliaça de resumo = 04

Fonte: A pesquisa, 2019. 


\section{ESTUDOS SELECIONADOS}

Foram analisados 20 artigos, apresentados de acordo com as suas características e critérios de inclusão e exclusão, determinados anteriormente.

Os dados estão dispostos em Tabelas (Tabela 1 e 2), levando em consideração, as principais informações obtidas e discutidas nesse estudo.

A Tabela 1 denota as características principais dos estudos, destacando: autor/ ano, tipo de estudo, base de dados e periódico.

Tabela 1 Características dos estudos incluídos.

\begin{tabular}{|c|c|c|c|}
\hline AUTOR, ANO & TIPO DE ESTUDO & $\begin{array}{l}\text { BASES DE } \\
\text { DADOS }\end{array}$ & PERIÓDICO \\
\hline SILVA, 2011 & $\begin{array}{c}\text { ESTUDO } \\
\text { EXPLORATÓRIO } \\
\text { QUALITATIVO }\end{array}$ & $\begin{array}{l}\text { GOOGLE } \\
\text { SCHOLAR }\end{array}$ & $\begin{array}{l}\text { UNIVERSIDADE } \\
\text { FEDERAL } \\
\text { FLUMINENSE }\end{array}$ \\
\hline BELEZA et al., 2012 & $\begin{array}{c}\text { ESTUDO } \\
\text { DESCRITIVO }\end{array}$ & BVS & REBEn \\
\hline LOPES et al., 2012 & $\begin{array}{c}\text { ESTUDO } \\
\text { DESCRITIVO } \\
\text { QUALITATIVO }\end{array}$ & BVS & $\begin{array}{l}\text { REV. DE PESQUISA: } \\
\text { CUIDADO É } \\
\text { FUNDAMENNTAL }\end{array}$ \\
\hline LIMA et al., 2013 & $\begin{array}{l}\text { REVISÃO DE } \\
\text { LITERATURA }\end{array}$ & $\begin{array}{l}\text { GOOGLE } \\
\text { SCHOLAR }\end{array}$ & $\begin{array}{l}\text { REISTA UNINGA } \\
\text { REVIEW }\end{array}$ \\
\hline COSTA et al., 2015 & $\begin{array}{l}\text { REVISÃO } \\
\text { INTEGRATIVA }\end{array}$ & $\begin{array}{l}\text { GOOGLE } \\
\text { SCHOLAR }\end{array}$ & $\begin{array}{c}\text { CARPE DIEM:REVISTA } \\
\text { CULTURAL E } \\
\text { CIENTIFICA DO } \\
\text { UNIFACEX }\end{array}$ \\
\hline DENGO et al., 2016 & $\begin{array}{c}\text { ESTUDO } \\
\text { QUALITATIVO E } \\
\text { DESCRITIVO } \\
\end{array}$ & BVS & COGITARE ENFERM. \\
\hline $\begin{array}{c}\text { GARRETT; } \\
\text { OSELAME; NEVES, } \\
2016 \\
\end{array}$ & $\begin{array}{c}\text { ESTUDO } \\
\text { DESCRITIVO } \\
\text { QUALITATIVO } \\
\end{array}$ & $\begin{array}{l}\text { GOOGLE } \\
\text { SCHOLAR }\end{array}$ & $\begin{array}{c}\text { REV. SAUDE E } \\
\text { PESQUISA MARINGA }\end{array}$ \\
\hline $\begin{array}{c}\text { MESEGUER et al., } \\
2016\end{array}$ & $\begin{array}{c}\text { ESTUDO } \\
\text { DESCRITIVO, } \\
\text { TRANSVERSAL E } \\
\text { ANALITICO } \\
\end{array}$ & BVS & RLAE \\
\hline VILLELA et al., 2016 & $\begin{array}{c}\text { ESTUDO } \\
\text { DESCRITIVO }\end{array}$ & BVS & $\begin{array}{l}\text { REV. ENFERMAGEM } \\
\text { UERJ }\end{array}$ \\
\hline
\end{tabular}




\section{QUANTIQUALITATIVO}

\begin{tabular}{|c|c|c|c|}
\hline $\begin{array}{l}\text { CAMBOIM et al., } \\
2017\end{array}$ & $\begin{array}{c}\text { PESQUISA } \\
\text { QUALITATIVA }\end{array}$ & $\begin{array}{l}\text { GOOGLE } \\
\text { SCHOLAR }\end{array}$ & ARQ. CIÊNC. SAÚDE. \\
\hline $\begin{array}{l}\text { POMPEU et al., } \\
2017\end{array}$ & $\begin{array}{c}\text { ESTUDO } \\
\text { DESCRITIVO } \\
\text { QUALITATIVO }\end{array}$ & BVS & RECOM \\
\hline $\begin{array}{l}\text { GUIMARÃES et al., } \\
2018\end{array}$ & $\begin{array}{c}\text { REVISÃO } \\
\text { INTEGRATIVA }\end{array}$ & $\begin{array}{l}\text { GOOGLE } \\
\text { SCHOLAR }\end{array}$ & $\begin{array}{l}\text { REV. ENFERMAGEM } \\
\text { UFPE ON LINE }\end{array}$ \\
\hline ROCHA et al., 2018 & $\begin{array}{c}\text { ESTUDO } \\
\text { DESCRITIVO E } \\
\text { RETROSPECTIVO }\end{array}$ & BVS & $\begin{array}{c}\text { COGITARE } \\
\text { ENFERMAGEM }\end{array}$ \\
\hline $\begin{array}{c}\text { ROCHA; } \\
\text { ZAMBERLAN, } 2018\end{array}$ & $\begin{array}{c}\text { REVISÃO } \\
\text { INTEGRATIVA }\end{array}$ & BVS & REVOL \\
\hline $\begin{array}{l}\text { ANDRADE et al., } \\
2019\end{array}$ & $\begin{array}{c}\text { REVISÃO } \\
\text { BIBLIOGRAFICA }\end{array}$ & $\begin{array}{l}\text { GOOGLE } \\
\text { SCHOLAR }\end{array}$ & GEP NEWS \\
\hline $\begin{array}{l}\text { CARNIEL et al., } \\
2019\end{array}$ & $\begin{array}{c}\text { REVISÃO } \\
\text { INTEGRATIVA }\end{array}$ & $\begin{array}{l}\text { GOOGLE } \\
\text { SCHOLAR }\end{array}$ & $\begin{array}{c}\text { JORNAL OF NURSING } \\
\text { AND HEALTH }\end{array}$ \\
\hline $\begin{array}{c}\text { DESSANTI; NUNES, } \\
2019\end{array}$ & $\begin{array}{c}\text { REVISÃO } \\
\text { BIBLIOGRAFICA }\end{array}$ & $\begin{array}{l}\text { GOOGLE } \\
\text { SCHOLAR }\end{array}$ & $\begin{array}{c}\text { REV. MEDICINA DE } \\
\text { FAMILIA E SAUDE } \\
\text { MENTAL }\end{array}$ \\
\hline $\begin{array}{l}\text { LINS; SILVA; } \\
\text { OLIVEIRA, } 2019\end{array}$ & $\begin{array}{c}\text { REVISÃO } \\
\text { INTEGRATIVA }\end{array}$ & $\begin{array}{l}\text { GOOGLE } \\
\text { SCHOLAR }\end{array}$ & CNPq \\
\hline $\begin{array}{c}\text { ROCHA; } \\
\text { WESTPHAL; } \\
\text { GOLDMAN, } 2019\end{array}$ & $\begin{array}{c}\text { ESTUDO } \\
\text { DESCRITIVO DE } \\
\text { CORTE } \\
\text { TRANSVERSAL }\end{array}$ & $\begin{array}{l}\text { GOOGLE } \\
\text { SCHOLAR }\end{array}$ & $\begin{array}{l}\text { BRASILIAN JOURNAL } \\
\text { O HEALTH REVIEW }\end{array}$ \\
\hline SILVA; SILVA, 2019 & $\begin{array}{c}\text { REVISÃO } \\
\text { INTEGRATIVA }\end{array}$ & $\begin{array}{l}\text { GOOGLE } \\
\text { SCHOLAR }\end{array}$ & GEP NEWS \\
\hline
\end{tabular}

Fonte: A pesquisa, 2019.

A apresentação da figura 2, inclui: autor/ano, resultados e discussão, e abrange os principais fatores associados a pratica da episiotomia. 
Tabela 2 Estudos selecionados e principais resultados.

AUTOR, ANO RESULTADOS E CONCLUSÕES

Os resultados apontam: o desconhecimento das mulheres em relação aos aspectos que norteiam e envolvem a episiotomia/rafia; uma visão distorcida do que vem a ser a episiotomia e de sua finalidade; ausência de orientações acerca do procedimento e dos cuidados necessários; perda de autonomia da mulher no processo de parto e nascimento e o fortalecimento da episiotomia/rafia como prática rotineira. As mulheres não guardam significado da episiotomia/rafia, pois os profissionais de saúde não se comprometem em preparar essas mulheres para o parto normal e SILVA, 2011 muito menos para a realização da episiotomia/rafia. É importante reforçar a necessidade de se rever os ensinamentos sobre a episiotomia/rafia no ensino obstétrico de enfermagem, onde deve ser feito a valorização das práticas não invasivas no processo do parto e nascimento ao invés da realização dessa intervenção. A enfermagem está presente na assistência geral e na específica de atuação no parto, por isso temos o dever de oferecer uma assistência de qualidade e dar condições para que ela possa lidar com as transformações que ocorrem em seu corpo no parto e no puerpério, proporcionando a ela um parto seguro, prazeroso e natural e um puerpério agradável e confiante dela mesma.

Para avaliação da dor foi utilizada a Escala Numérica e o Questionário McGill, bem como um formulário para analisar as atividades que estavam limitadas. A média de dor perineal BELEZA et al., encontrada foi cinco. As categorias sensorial e avaliação subjetiva 2012 foram as mais selecionadas no Questionário McGill. A dor perineal foi caracterizada como latejante, que repuxa, que esquenta, ardida, dolorida, chata, incômoda, que prende e que deixa tensa. Sentar, deitar e deambular foram as atividades mais limitadas.

O material coletado apontou que apesar das puérperas referirem que aceitam o procedimento por que ele facilita o parto, abreviando LOPES et al., 2012 o sofrimento materno e fetal, elas vivenciam sentimentos de dúvidas, medos e diversas repercussões no puerpério imediato, dentre elas, a dor associada ao corte e a episiorrafia, assim como incômodos nas realizações de atividades, as quais: deambulação, eliminações vesicais e intestinais e amamentação.

Pode-se afirmar que a episiotomia tornou-se rotineira, usada de modo profilático como justificativa para evitar traumas perineais à morbidade e mortalidade infantil e problemas ginecológicos tais LIMA et al., como retocele, cistocele e relaxamento do assoalho pélvico. $O$ 2013 retorno às atividades sexuais varia num tempo específico para cada mulher dependendo da sua libido, do "medo", da cicatrização das incisões ou lacerações perineais e do grau de atrofia vaginal secundária e a episiorrafia é um evento que interfere nesse tempo. 

\begin{tabular}{ll}
\hline & Muitas mulheres não participam da decisão de se fazer ou não a \\
& episiotomia nem tão pouco são informadas a respeito das práticas \\
& rotineiras que trazem consequência e influenciam na qualidade da \\
& vida sexual da mesma. \\
& A pesquisa revela grande incidência da episiotomia no momento do \\
parto normal, chegando a $90 \%$ e destaca como complicações da \\
COSTA et al., \\
epsisiotomia o aumento de hemorragia pós parto, dor no período \\
puerperal, maior tempo de internamento, formação de hematomas, \\
incontinência urinária e fecal, formação de fístulas e dispareunia. \\
\hline Os dados foram analisados com base na análise temática, \\
emergindo os seguintes temas: Episiotomia: o conhecido \\
desconhecido; "Cortando" o direito de escolha; Desconhecendo as \\
consequências da episiotomia. Observou-se que as mulheres não \\
co16 et al., \\
são informadas e orientadas a respeito da realização da episiotomia, \\
o que leva ao entendimento errôneo sobre esta prática e à limitação \\
do direito de escolha da mulher. Isso indica a necessidade de \\
ampliação do conhecimento e do resgate da autonomia da mulher \\
no processo de parto e nascimento.
\end{tabular}

Em $76,27 \% \quad(n=45)$ foram submetidas à episotomia sem 0 consentimento ou conhecimento prévio. Emergiram durante a entrevista fatores como grau de conhecimento e de orientação antecipada sobre o procedimento; possíveis complicações e GARRETT; cuidados após o parto; reação emocional e equipe de saúde frente a OSELAME; tal situação. As parturientes que só realizaram partos normais em NEVES, 2016 todas as gestações predominaram com $52 \%(n=26)$ com faixa etária de 18 a 25 anos. Observou-se uma notória insatisfação das entrevistadas enquanto pacientes. As falas sugerem que a realização da episiotomia representou uma violação dos direitos sexuais, reprodutivos e emocionais das mesmas.

A taxa de episiotomia encontra-se acima das recomendações da OMS, que constatou que a episiotomia não é um procedimento isolado e independente de outras práticas obstétricas. Em vez disso, está associada à prática de determinadas variáveis clínicas que influenciam o aumento na taxa desse procedimento. A porcentagem global de episiotomias foi de $50 \%$. As variáveis clínicas que MESEGUER et apresentaram uma associação significativa foram: primiparidade al., $2016 \quad(R R=2,98)$, idade gestacional $>41$ semanas $(R R=1,2)$, início do parto estimulado ou induzido $(R R=1,33)$, uso de analgesia epidural $(R R=1,95)$, uso de ocitocina $(R R=1,58)$, posição de litotomia durante a expulsão fetal $(R R=6,4)$ e instrumentação $(R R=1,84)$. Por outro lado, idade materna $\geq 35$ anos $(R R=0.85)$ e peso do neonato $<2500$ g $(R R=0,8)$ estão associados a uma menor incidência de episiotomia.

Evidenciaram déficit quanto ao esclarecimento das mulheres sobre a VILLELA et al., episiotomia e repercussão dos sentimentos vivenciados pelas 2016

mulheres no puerpério. As episiotomias foram realizadas sem informação e sem autorização prévia. 
Quando questionadas quanto ao conhecimento da episiotomia, todas as mulheres relataram não terem conhecimento sobre esse assunto. Comprovou-se como principais alterações emocionais, o CAMBOIM et medo de realizar algum esforço físico em função da presença da al., 2017 episiorrafia. Como alterações físicas, as participantes do estudo apontaram dor, dificuldade de locomoção no leito, ardência, prurido, dificuldade para micção e evacuação, e dispareunia como principais incômodos físicos.

Apontam para a falta de esclarecimento e o desconhecimento das participantes quanto ao termo episiotomia, fatores que podem POMPEU et al.,influenciar o evento do parto e, ainda, a violência de gênero que 2017 ocorre nas instituições de saúde, como a violência obstétrica, a qual está perpetrada nas maternidades e, muitas vezes, não é percebida por quem as pratica e, também, por quem sofre essa violência.

GUIMARÃES Foi possível verificar que a maioria dos estudos se referem à prática et al., 2018 da episiotomia como intimamente ligada a primiparidade, rigidez perineal, macrossomia e prematuridade.

A episiotomia ocorreu em 174 (19,7\%) partos e em 512 (59\%) houve lacerações perineais. A integridade perineal foi mantida em 187 $(21,4 \%)$ partos. As principais indicações estiveram relacionadas às condições do períneo: 54 (58,1\%) por rigidez perineal, 22 (23,7\%) ROCHA et al., períneo curto e 19 (20,4\%) eminência de laceração grave. 2018 Conclusão: a prática de episiotomia entre residentes está acima do recomendado pela OMS e, com relação às justificativas apresentadas, há discrepância com as mundialmente Utilizadas, trazendo reflexões acerca do modelo de formação e do distanciamento entre teoria e prática.

Emergiram as categorias: Fatores relacionados à prática da ROCHA; $\quad$ episiotomia e ocorrência de lacerações perineais e indicações para a ZAMBERLAN, realização; Abordagem do profissional que presta assistência ao 2018 parto normal e Estratégias para a prevenção de lacerações perineais.

Os enfermeiros tem uma relevância ao que se diz sobre práticas humanizadas na obstetrícia, por isso vale ressaltar que é preciso

ANDRADE et al., 2019 além de uma padronização de métodos e proibições de alguns métodos considerados violentos é necessário que os profissionais se adéquem as práticas que tragam benefícios deixando as que estão em desusos para que se evitem os efeitos negativos nas parturientes.

Amostra composta por 31 artigos, dos quais 22 (71\%) foram escritos em português e nove $(29 \%)$ em inglês. A violência obstétrica é definida como atos e procedimentos que prejudicam a mulher nos CARNIEL et al aspectos físicos, verbais e psicológicos em todo o ciclo gravídico2019 puerperal. A episiotomia quando realizada de forma rotineira e sem autorização da mulher configura-se violência obstétrica. Os estudos analisados nesta revisão procuram transformar 0 modelo intervencionista em humanizado e que mantenham a autonomia da mulher no trabalho de parto, com uma assistência fundamentada 
cientificamente.

O Ministério da Saúde do Brasil, apesar de recomendar o seu uso seletivo, não determina a taxa ideal a ser atingida. Estima-se, por alguns autores, que uma frequência ótima deveria situar-se entre 10 a $30 \%$ do total de partos vaginais. A técnica não impede o aparecimento da dispareunia ou melhora a função sexual da paciente no período pós-parto. Muitos autores associam a episiotomia a um nível maior de dor e que não há diferença DESSANTI; significativa entre o aparecimento de incontinência urinária nas NUNES, 2019 mulheres submetidas ou não a episiotomia. De modo que a aplicação rotineira dessa técnica não diminui a dor ou previne incontinência urinária. Nenhuma pesquisa de qualidade adequada demonstrou que a episiotomia reduziria o risco de laceração retal ou diminuiria o tempo de cicatrização. Nenhum estudo apoiou a episiotomia rotineira como uma intervenção protetora para incontinência anal. Esse procedimento não teve efeito protetor nem causador sobre o prolapso urogenital.

LINS; SILVA; Observou-se além de pouco conhecimento e esclarecimento, não foi OLIVEIRA, solicitado a autorização para realização da episiotomia, tendo como 2019 consequência física e emocional de curto a longo prazo.

Evidenciou-se que as participantes relataram conhecimento "muito

ROCHA; bom" em relação a episiotomia e indicam a realização na existência WESTPHAL; de feto macrossômico, em iminência de rotura perineal, na presença GOLDMAN, de mecônio, na distócia de ombro, na primariedade e prematuridade. 2019 No entanto, os achados apontam a existência de desafios no conhecimento que necessitam ser superados.

A atuação da enfermagem é um fator de grande relevância para o estímulo ao parto fisiológico e humanizado onde a mulher se torna a protagonista deste momento natural, executando uma diminuição das influências externas que direcionam para um parto com SILVA; SILVA, intervenções. As ações a serem realizadas pela enfermagem 2019 incluem a promoção de um parto mais humanizado, promoção de uma capacitação aos profissionais que prestam atendimento às parturientes, como também ações educativas durante o pré-natal, tornando a mulher protagonista do processo do parto, desta maneira prevenindo ou diminuindo a violência obstétrica.

Fonte: A pesquisa, 2019.

\section{HISTORICIDADE DO PARTO E EPISIOTOMIA}

Os costumes e os métodos relacionados ao parto tem se descaracterizado ao longo dos tempos, todavia é de grande relevância, promover um parto e nascimento 
saudável, em um momento que é tão significativo na vida da mulher. O parto é um processo fisiológico que tem início e evolução natural, acompanhadas por contrações uterinas, até que ocorra entre 2 a $10 \mathrm{~cm}$ de dilatação do colo do útero, proporcionando a expulsão do feto, que pode acontecer de maneira saudável, segura e sem intervenções médicas (COSTA et al., 2015).

Neste momento, as mulheres podem ser submetidas a procedimentos invasivos e que podem ser prejudicial, a exemplo da episiotomia, uma incisão cirúrgica, realizada no período expulsivo, na região da vulva (CAMBOIM et al., 2017).

A episiotomia foi inicialmente defendida por Pomeroy (1918) e De Lee (1920) como uso profilático, a fim de evitar traumas e complicações fetais, e por durante muitos anos, esta prática foi bem aceita e largamente difundida, ainda que sem embasamento científico que comprovasse a sua eficácia (GUIMARÃES et al., 2018).

Outro processo, que colaborou para a sustentação desse modelo se deu pelo interesse crescente da medicina na obstetrícia associado à presença de homens auxiliando no trabalho de parto a partir do século XVIII, o que contribuiu para um aumento do índice de mortalidade. A mulher deixa o ambiente domiciliar e passa a utilizar o ambiente hospitalar, tornando o parto medicalizado (OLIVEIRA; MIQUILINI, 2005).

Refletindo sobre o paradigma vigente da época, a prática era habitualmente justificada, como prevenção de trauma perineal severo, danos do assoalho pélvico, de prolapsos e incontinências urinárias, além de ser considerada uma incisão limpa e regular (GUIMARÃES et al., 2018). Entretanto, a prática da episiotomia, tornou-se um procedimento generalista e rotineiro, realizada de forma indiscriminada e que gera diversas repercussões na vida da mulher (LOPES et al.,2012).

A episiotomia pode ser do tipo médio-lateral, mediana ou lateral, objetivando aumentar o diâmetro de saída do canal de parto. A incisão médiolateral abrange a pele, mucosa vaginal, aponeurose superficial do períneo e fibras. A incisão mediana apresenta maiores vantagens quando comparados às outras incisões, e a que mais preserva a integridade anatômica e causa menos sofrimento a mulher. A incisão lateral, por sua vez não é mais praticada, em virtude da vascularização do local (LIMA et al., 2013). 
As mulheres são induzidas, por alguns profissionais da saúde, de que seus corpos são incapazes de parir naturalmente e que existe a necessidade da ajuda dos profissionais e da prática da episiotomia para possibilitar a passagem do feto na hora do parto, evitando assim, maiores riscos. Para tal afirmativa, faz necessário destacar a importância das mulheres ser conhecedora do próprio corpo e de seus direitos (DENGO et al., 2016).

A episiotomia é considerado uma violência obstétrica, onde o cuidado ofertado por profissionais obstétricos, infringe o direito da mulher, por meio de maus tratos físicos, psicológicos e verbais, com procedimentos danosos a saúde (GARRETT; OSELAME; NEVES, 2016).

\section{EVIDÊNCIAS CIENTÍFICAS CONTRA EPISIOTOMIA}

Não existem evidências científicas da beneficência da prática recorrente da episiotomia, destacando a necessidade de transformar o modelo intervencionista em uma ideologia humanizada (CARNIEL; VITAL; SOUZA, 2019). Foram identificados que os fatores mais associados ao uso rotineiro da episiotomia são primiparidade, rigidez perineal, macrossomia e prematuridade, o que difere a organização mundial de saúde (OMS) e o ministério da saúde (MS), que contraria o uso rotineiro e liberal da episiotomia, utilizado de modo assistemático (GUIMARÃES et al., 2018).

Estudos apontam uma relação significante entre episiotomia e o aumento do risco de lacerações graves, de $3^{\circ}$ e $4^{\circ}$ grau, com lesões de esfíncter anal, indicando que esta prática deve ser desestimulada. Não há evidências de que a episiotomia previne lacerações graves ou relaxamento do pavimento pélvico, mas admite-se que esta prática desenvolva um maior risco de traumatismo do períneo e complicações, tais como, o aumento de hemorragias pós-parto, extensão da incisão, dor pós-parto, disfunção sexual, infecções e deiscência, formação de hematomas e dispaurenia (ROCHA; WESTPHAL; GOLDMAN, 2019).

A situação descrita acima é reafirmada por Figueredo et al (2011) e Costa et al (2011) e apontam diversas complicações relacionadas a episiotomia como a perda 
sanguínea aumentada quando comparado a perda sanguínea do parto sem episiotomia, infecção, disfunção sexual como dispaurenia, incontinência urinaria, prolapso uterino, extensão da lesão perineal, hemorragia, edema, hematomas, fístulas retovaginais, endometriose presente na cicatriz, lesão do tecido muscular, nervoso, mucosa e pele.

Mulheres episiotomizadas manifestam sentimentos de medo, que acarreta dor física, angustias e expectativas geradas em torno da episiotomia, o que infringe os direitos sexuais da mulher, sua integridade física e sexual, desvalorizando suas opiniões, os anseios e medos acarretados por este procedimento (LOPES et al., 2012).

Atualmente, estudos evidenciam o desuso da episiotomia, apontando a técnica como inadequada e que só deve ser usadas em casos específicos. É esperado que os enfermeiros prestem uma assistência humanizada, que vise preservar a fisiologia do parto, evitando assim, intervenções desnecessárias (GUIMARÃES et al., 2018).

\section{HUMANIZAÇÃO DAS PRÁTICAS DE ENFERMAGEM}

Os estudos apontam a carência de conhecimentos das mulheres, quando questionadas sobre a episiotomia. Há um desprovimento de informação, antes, durante e após o processo de parto e nascimento, por parte dos profissionais de saúde, impedindo um atendimento contextualizado e qualificado (CAMBOIM et al., 2017).

A mulher deve ser informada e orientada durante o pré-natal sobre o que é e porque é utilizado tal procedimento e os profissionais da saúde devem ser devidamente treinados e atualizados acerca das diretrizes baseadas nas evidências a fim de contribuir com a diminuição das taxas desnecessária de episiotomia e suas consequências nocivas à saúde física e psíquica da mulher (POMPEU et al., 2017).

O Ministério da Saúde (MS) encoraja a atuação da enfermagem no pré-natal e no parto, focada na humanização da assistência, evitando intercorrências e 
respeitando a fisiologia do parto. Propõe a valorização da informação, de forma a viabilizar o empoderamento e a autonomia das mulheres, para que esta possa vivenciar a gestação, o parto e pós parto em sua plenitude (DENGO et al., 2016).

Compete aos profissionais de enfermagem, obstetras e obstetrizes buscar atualizar os conhecimentos e basear suas práticas em evidências científicas, afim de analisar a necessidade de realizar a episiotomia, e aplicar técnicas seguras de modo a reduzir o uso liberal desta prática (ROCHA; WESTPHAL; GOLDMAN, 2019).

A enfermagem desenvolve um papel primordial na transformação dos serviços de saúde ofertados as mulheres durante o processo gravídico-puerperal, principalmente como educador em saúde, uma vez que é detentor do conhecimento, fundamentada na prevenção e promoção da saúde da mulher (VILLELA et al., 2016).

Incube aos profissionais de enfermagem realizar procedimentos que podem reduzir ou evitar a prática da episiotomia, por meios de exercícios para fortalecer o períneo ou adotar uma posição não horizontal, com o objetivo de evitar lacerações (POMPEU et al., 2017).

Se faz necessário ainda, orientações e execução de métodos não farmacológicos, no qual podemos citar, o uso de bolas suíças, massagens que proporcionam maior conforto, o uso do balanço pélvico e banhos mornos, de forma a viabilizar a participação ativa da mulher (COSTA et al., 2015).

O enfermeiro é indispensável no processo de humanização, como descreve Costa et al (2015) que diante deste processo complexo, e com mudanças peculiares na região genital feminina, surge à necessidade de uma assistência de qualidade, que visa à reintegração do aparelho reprodutivo feminino. Nesse contexto, destacase a importância do enfermeiro obstetra na condução do processo de TP e dos demais membros da equipe de enfermagem nos cuidados a mulher de pós-parto normal submetida à episiotomia.

O cuidado deve ser centrado nas necessidades individuais de cada mulher. $O$ enfermeiro deve estar apto a orientar, esclarecer dúvidas, acolher, aconselhar e auxiliar no parto. Manter uma comunicação ativa com a paciente, desenvolve vínculos de confiança, de modo a garantir a humanização na assistência de enfermagem (POMPEU et al, 2017). 


\section{CONSIDERAÇÕES FINAIS}

A prática da episiotomia segundo a Organização Mundial da Saúde (OMS) continua muito acima do permitido, sabe-se que esta prática deve ser restritamente reservada para casos específicos, que possa comprometer a saúde e segurança da mulher no momento do parto. Muitas mulheres são episiotomizadas sem nenhuma indicação, levando a sérios riscos de saúde como Lesões nos músculos da região íntima, Incontinência urinária, Infecção no local do corte, Aumento do tempo de recuperação pós-parto e até mesmo trauma de ter relações sexuais e engravidar novamente.

Diante disso, se faz necessário dar visibilidade e autonomia, ampliando a visão ativista da mulher, de maneira a decidir sobre qualquer procedimento em relação ao seu corpo. Os princípios que norteiam a assistência humanizada está no protagonismo da mulher e no respeito de suas escolhas, e não de impor interferências em um momento de tanta vulnerabilidade.

A educação e atualização dos conhecimentos, a inclusão da equipe de enfermagem no cuidado ao parto, a adoção de boas práticas e a redução das intervenções durante o parto resultam em um número reduzido de episiotomia e melhor qualidade de vida após o processo parturitivo. 


\section{REFERÊNCIAS BIBLIOGRÁFICAS}

ARATANI, N. et al. Preferência do tipo de parto entre gestantes primíparas. Revista de Odontologia (ATO), Bauru/SP, ed. 14, n. 3, p. 209-224, 2014. Disponível em http://www.actiradentes.com.br/revista/2014/textos/12revistaATOGestante_primiparas-

2014.pdf. Acesso em 22 mar. 2019.

ANDRADE, V. B. et al. Efeitos da violência obstétrica causados as gestantes no parto e posparto: $E$ a humanização da assistência de enfermagem. GEPNEWS, Maceió, a.3, v.2, n.2, p.6974, abr./ jun. 2019.

BELEZA, A.C.S. et al. Mensuração e caracterização da dor após episiotomia e sua relação com a limitação de atividades. Rev Bras Enferm, Brasília 2012 MarAbr, 65(2):264-8. Disponível em: http://dx.doi.org/10.1590/S0034-71672012000200010. Acesso em 18 Abr. 2019.

BRASIL. Ministério da Saúde. Política Nacional de Atenção Obstétrica e Neonatal. Portaria n 1.067/ GM de 4 de Julho de 2005. Acesso em 18 Mar. 2019.

CAMBOIM, F.F. et al. História oral de vida temática de mulheres em relação a episiotomia. Arq. Ciênc. Saúde, 24(2) 25-32, abr./jun. 2017.

CARNIEL, F; VITAL, D.S; SOUZA, T.D.P. Episiotomia de rotina: necessidade versus violência obstétrica. J. nurs. Health. 2019; 9(2): e199204.

COSTA, M.L. et al. Episiotomia no parto normal: Incidência e complicações. Carpe Diem: revista cultural e cientifica do UNIFACEX, V. 13, n.1, 2015. ISSN: 2237-8685.

COSTA, N.M. et al. Episiotomia nos partos normais: uma revisão de literatura. Revista Facene/Famene, Mossoró/RN, v.9, n. 2, p. 45-50, 2011. Disponível em http://www.facene.com.br/wp-content/uploads/2010/11/2011-2-pag-45-50. Episiotomia.pdf. Acesso em 26 mar. 2019.

DENGO, V.A.R. et al. A episiotomia na percepção de puérperas. Cogitare enferm. 2016 jul/set, 21(3): 01-08.

DESSANTI, G.A; NUNES, C.P. complicações e sintomas no pos-parto com episiotomia. Revista de Medicina de Família e Saúde Mental. V.1, N. 01, 2019.

FIGUEIREDO, G.S. et al. Ocorrência de episiotomia em partos acompanhados por enfermeiros obstetras em ambiente hospitalar. Revista Enfermagem UERJ, Rio de Janeiro, v. 19, n. 2, p. 181-185, 2011 abr./jun. Disponivel em http://www.facenf.uerj.br/v19n2/v19n2a02.pdf. Acesso em 16 mai. 2019.

GARRETT, C.A; OSELAME, G.B; NEVES, E.B. O uso da episiotomia no sistema único de saúde brasileiro: a percepção das parturientes. Revista Saúde e pesquisa, Maringá. V.9, n.3, p.453-459, set./dez. 2016. ISSN 1983-1970- e- ISSN 2176-9206. Disponível em http://dx.doi.org/10.177651/1983-1970.2016v9n3p453- 459. Acesso em 12 ago. 2019.

GOMES, A.R.M. et al. Assistência de enfermagem obstétrica na humanização do parto normal. Revista cientifica de Enfermagem, v. 4, n. 11, p.23-27, 2014. Disponível em: http://www.recien.com.br/online/index.php/Recien/article/view/73.Acesso em: 22 mar. 2019.

GUIMARAES, N.N.A. et al. Analise de fatores associados a pratica da episiotomia. Revista de enfermagem UFPE online, Recife,12(4):1046-53, abr. 2018. 
LIMA, M.G. et al. A episiotomia e o retorno a vida sexual pos-parto. Revista Uningá review. V.16, n.2, p.33-37, out./dez.2013. ISSN 2178-25571.

LINS, H.C. et al. Violência obstétrica: percepção das puérperas frente a episiotomia. CNPq, 2019.

LOPES, D.M. et al. Episiotomia: sentimentos e repercussões vivenciadas pelas puérperas. Revista de Pesquisa: cuidado é fundamental, v. 4, n. 1, p. 2623-2635, 2012.

MATHIAS, A.E.R.A. et al. Mensuração da dor perineal no pós-parto vaginal imediato. Rev. Dor, São Paulo, v. 16(4):267-71, Out./dez. 2015. Disponível em http://dx.doi.org/10.5935/18060013.20150054. Acesso em 2019 Mar 20.

MESEGUER, C.B. et al. Episiotomia e sua relação com diferentes variáveis clinicas que influenciam sua realização. Rev. Latino- Am. Enfermagem. 2016, 24: e2793. Disponível em http://dx.doi.org/10.1590/1518-8345. 0334.2686. Aceso em 12 ago. 2019.

MINISTÉRIO DA SAÚDE, Universidade Estadual do Ceará. Caderno Humaniza sus. Humanização do parto e do nascimento. Brasília (DF): Ministério da Saúde; 2014. Disponível em:http://www.redehumanizasus.net/sites/default/files/caderno_humanizasus_v4_humanizaca o_parto.pdf. Acesso em 20 mar. 2019.

OLIVEIRA, S. M. J. V; MIQUILINI, E. C. Frequência e critérios para indicar a episiotomia. Rev. Esc. Enferm. USP, v. 39, n.3, p. 288-95, 2005.

ORGANIZAÇÃO MUNDIAL DA SAÚDE (OMS). Maternidade segura - Assistência ao parto normal: um guia prático. Genebra: OMS; 1996.

PINHEIRO, A. et al. Cartilha pelo direito ao parto normal: um visão partilhada. Ed maio. Portugal/Lisboa. Associação Portuguesa de Enfermeiros Obstetras/Ordem dos enfermeiros, 2012.

http://www.ordemenfermeiros.pt/publicacoes/documentos/livro parto normal.pdf. Acesso em: 24 mar. 2019.

POMPEU, K.C. et al. Pratica da episiotomia no parto: desafios para a enfermagem. Revista de enfermagem do centro-oeste mineiro. 2017; 7:e1142. Disponível em: http://dx.doi.org/10.19175/recom.v7i0.1142. Acesso em 12 ago. 2019.

ROCHA, B.D; ZAMBERLAN, C. Prevenção de lacerações perineais e episiotomia: evidencias para a pratica clínica. Rev enferm UFPE, Recife, 12(2):489-98, fev. 2018.

ROCHA, C.A; WESTPHAL, F; GOLDMAN, R.E; Conhecimento, atitude e pratica de enfermeiros obstetras e obstetrizes sobre a realização da episiotomia. Braz. J. Hea.,Curitiba, v.2,n.1,p.226235, jan./feb.2019. Disponível em: http://www.brjd.com.br/index.php/BJHR/article/view/903. Acesso em: 19 mar. 2019.

ROCHA, E.S. et al. Pratica de episiotomia entre residentes em enfermagem obstétrica. Cogitare enferm, (23) 4:e5445, 2018.

SALGE, A.K.M. et al. Prática da Episiotomia e Fatores Maternos e Neonatais Relacionados. Rev. Enf., $2012, \quad 14 \quad(4): 779-85 . \quad$ Disponivel em: https://www.fen.ufg.br/fen_revista/v14/n4/pdf/v14n4a05.pdf. Acesso em 18 abr. 2019.

SILVA, L.F. A episiotomia/rafia na percepção das mulheres: A enfermagem em busca da melhoria do cuidar. Trabalho de conclusão de curso. Universidade federal fluminense. Niterói, 2011. 64f. 
SILVA, E.H.B; SILVA, J.N.D. Ações de enfermagem capazes de prevenir ou diminuir a violência obstétrica: revisão integrativa. GEPNEW, Maceió, a.3, v.2, n.2, p.589-598, abr./jun. 2019.

VILLELA, J.P. et al. Episiotomia: sentimentos vivenciados pelas puérperas. Rev enferm UERJ, RIO DE JANEIRO, 2016; 24(5):e21882. Disponível em https://dx.doi.org/10.12957/reuerj. 2016.21882. Acesso em 22 mai. 2019. 